\title{
Uso de aplicaciones móviles educativas para niños con dificultades de aprendizaje
}

Use of educational mobile applications for children with learning difficulties

Recibido: abril 11 de 2017 | Revisado: mayo 10 de 2017 | Aceptado: junio 15 de 2017

\author{
Ana Quispe ${ }^{1}$ \\ Carlos Bernal ${ }^{2}$ \\ Gilva SALAZAR ${ }^{3}$
}

\section{Resumen}

En la presente investigación, hacemos una evaluación de diferentes aplicaciones móviles educativas que apoyan a niños con dificultades de aprendizaje como Trastorno por Déficit de Atención e Hiperactividad (TDAH), dislexia, discalculia, disgrafía y dispraxia; corroborando que en nuestro país serían de mucha utilidad como apoyo a la enseñanza. Asimismo, confirmamos que las innovaciones de la gamificación facilitan a que los niños aprendan de una forma más divertida y genera, en ellos, una experiencia positiva.

Palabras clave: aplicaciones móviles, dificultades de aprendizaje, gamificación

\begin{abstract}
In the present research, we make an evaluation of different educational mobile applications that support children with learning difficulties such as Attention Deficit Disorder and Hyperactivity (ADHD), Dyslexia, Dyscalculia, Dysgraphia and Dyspraxia; corroborating that they would be very useful in our country as a support for teaching. We also confirm that the innovations of gamification make it easier for children to learn in a more fun way, thus generating in them a positive experience.
\end{abstract}

Keywords: mobile applications, learning difficulties, gamification

1 Universidad de San Martín de Porres, aquisper@usmp.pe

2 Universidad de San Martín de Porres, cbernalo@usmp.pe

3 Universidad de San Martín de Porres, gsalazarr@usmp.pe 


\section{Introducción}

Desde comienzos del siglo pasado, se han definido diferentes generaciones que fueron parte de un tiempo en la historia, según se menciona, en el libro Conectados en el Ciberespacio: Generación Interbellum, Generación Grandiosa, Generación Silenciosa, Baby Boomers, Generación X, Generación $Y$ y Generación $Z$. Como nuestro estudio está enfocado en niños, explicaremos con mayor detalle sobre la Generación Z o también llamada generación de Nativos Digitales (Aparicie, 2013).

Actualmente, la generación de nativos digitales tiene a disposición sistemas de información que potencian y complementan el conocimiento aprendido en clase como aplicativos para PCs, laptops y dispositivos móviles. También existen tecnologías orientadas a niños con problemas específicos las que los apoyan a desarrollar y mejorar sus capacidades que van a ser evaluadas en el presente estudio.

En la década del sesenta, padres y educadores debatían sobre la variedad de etiquetas con las que diagnosticaban a los niños con problemas específicos de aprendizaje como daño cerebral, disfunción cerebral mínima o problemas perceptivos (Aguilera, 2003). Pero no fue hasta el año 1963 en que Samuel Kirk plantea el término "learning disabilities" o en español, "dificultades de aprendizaje", concepto que englobaba alteraciones o retrasos en el desarrollo de uno o más de los procesos de lenguaje, habla, deletreo, escritura o aritmética, producto de una disfunción cerebral y/o trastorno emocional o conductual, desligando a esto problemas de retraso mental, de privación sensorial, factores culturales o instruccionales (Kirk, 1962).

En el presente artículo, abordamos los beneficios que tienen las aplicaciones móviles educativas para los niños, tomando como base la tendencia cada vez mayor, en nuestro país, del uso de las Tablet y Smartphones y la necesidad de apoyar a aquellos que tienen dificultades de aprendizaje como Trastorno por Déficit de Atención e Hiperactividad (TDAH), dislexia, discalculia, disgrafía y dispraxia.

\section{Dificultades a aprendizaje y de atención en niños}

El desarrollo infantil es un proceso dinámico, sumamente complejo, que se sustenta en la evolución biológica, psicológica y social. Las primeras etapas del desarrollo humano, comprendidas entre la infancia y la niñez (hasta los 12 ańos), constituyen una etapa de la existencia crítica ya que en ella se van a configurar las habilidades perceptivas, motrices, cognitivas, lingüísticas $y$ sociales que posibilitarán una equilibrada interacción con el mundo circundante (Federación Estatal de Asociaciones de Profesionales de AtenciónTemprana, 2000). Complementando este concepto, (Millá, 2006) asegura que en la infancia se logra una gran cantidad de aprendizajes que constituirán la base sobre la que se podrá conseguir el dominio de la lectura, la escritura y las matemáticas, entre otros.

Sin embargo, es posible que en esta etapa del desarrollo infantil, niños con una inteligencia normal, que crecen en un ambiente saludable y con una adecuada educación no logren aprender como el 
resto de infantes de su misma edad. Este retraso puede indicar la presencia de dificultades tempranas de aprendizaje, usualmente detectadas en el ámbito escolar, que podrían manifestarse de diferentes maneras y con diversos grados de severidad como problemas con las habilidades motoras, de socialización, de lenguaje, dificultades atencionales y perceptivas y limitaciones cognitivas o emocionales (Grupo de Atención Temprana, 2000) repercutiendo no solo en el ámbito académico, sino también, en el ámbito emocional, como mencionan Mainieri, A. \& Méndez, Z. (1986), un niño con problemas de aprendizaje necesita todo el apoyo estable y externo para que desarrolle un ego fuerte. Los constantes fracasos escolares pueden acarrear problemas de baja autoestima o rebeldía en otros casos. Por ello es importante actuar de inmediato tras la detección de estas deficiencias de aprendizaje con programas de acción para llevarlos hasta los límites de su capacidad de aprendizaje y adaptación (Mainieri \& Méndez, 1986).

Los tipos más comunes de dificultades son:

\section{a) Trastorno por Déficit de Atención e Hiperactividad (TDAH)}

Los niños con trastorno por déficit de atención e hiperactividad (TDAH) presentan, entre otras dificultades, problemas para realizar tareas relacionadas con el proceso de enfocar y ejecutar eficientemente, manejar dos sistemas de información, simultáneamente, así como problemas con las funciones ejecutivas y la capacidad de cambiar la atención adaptativamente, resistir la tendencia hacia la perseverancia y tener una memoria de trabajo eficiente (F. Quintero, Correas \& Quintero, 2009).

El síndrome se caracterizaría por una mórbida incapacidad para mantener la atención, por una falta de voluntad o de autocontrol (insuficiencia de inhibición volitiva), inquietud e impulsividad (excitabilidad exagerada) ańadido a una leve sintomatología neurológica marcada por la torpeza y anomalías congénitas menores (Taylor \& Rutter, 2002).

\section{b) Dislexia - Dificultad para leer}

Se entiende la dislexia como una amplia gama de manifestaciones que provocan dificultades significativas en las tareas de lectura y escritura. La dislexia ha sido especialmente evidente una vez que se han instaurado los sistemas universales de enseñanza. Es, por tanto, responsable de buena parte del fracaso escolar (Coveñas \& Sánchez, 2010). Mientras tanto (Torras, 2002) menciona que hay variaciones en la severidad del problema, desde niños que no consiguen aprender letras, hasta los que aprenden a leer, pero no logran comprender lo que leen, o consiguen escribir, pero no automatizan el grafismo ni la ortografía. La investigación teórica y empírica sobre la dislexia destaca la importancia del uso del color y del tacto en varios procesos perceptivos que pueden estar relacionados con la lectura. La dificultad para aprender a leer está relacionada con factores emocionales, sociales y económicos (Alissa, Antle \&Cramer, 2015).

\section{c) Discalculia - Problemas con las matemáticas}

Es una discapacidad específica del aprendizaje en matemáticas. Los nińos con 
discalculia tienen dificultades, en un gran número, en tareas aritméticas y numéricas, que involucran una gran cantidad de capacidades como organización espacial, atención visual, motricidad, juicio, razonamiento y memoria (Ardila, Rosselli $\&$ Matute, 2005). Algunos niños son incapaces de reconocer los números o de escribirlos o pueden tener problemas al contar. También presentan dificultad en comprender y realizar operaciones básicas como la suma, resta, la multiplicación y la división (Moore \& Jefferson, 2005).

\section{d) Disgrafía. Problemas para escribir}

Los niños con disgrafía del desarrollo, también denominada trastorno de la expresión escrita, comprenden lo que se les dice, hablan y leen claramente, pero tienen dificultades para el aprendizaje de la escritura. El defecto no está relacionado con la escritura manual ni con el dominio del bolígrafo, sino con los signos de puntuación, el deletreo de las palabras y la construcción global de las frases y oraciones (Moore, 2005).

\section{e) Dispraxia. Problemas con las habilidades motoras}

Las personas con dispraxia presentan dificultades en la planificación motora, lo que se manifiesta como torpeza (Kielhofner, 2004). En la nińez, se presenta como dificultades en el desempeño de tareas ordinarias, en sí, problemas que afectan las habilidades motoras gruesas como saltar, correr, mantener el equilibrio y también pueden afectar las habilidades motoras finas como sostener cosas, movimientos de la mano que se usan para escribir, movimientos de boca y lengua que se usan para hablar.

\section{Recursos Web Educativos para nińos con dificultades de aprendizaje}

Actualmente, podemos ver un avance en tecnología y sistemas de información, que cada vez está más al alcance de la población, los niños tienen más acceso a ella tanto en la escuela como en casa. La cada vez mayor audiencia infantil ha llevado a la creación de diferentes aplicaciones para Smartphone y Tablet, desde juegos para distracción como aquellos que tienen el objetivo de servir como apoyo a la enseñanza, por ejemplo, para ayudar a leer como "El Alfabeto en Español", en donde los niños relacionan una letra a cada objeto y así aprenden las letras del abecedario, "Kid's socks" para aprender a diferenciar los patrones, los colores y relacionarlos, "Where's my Water?", donde ofrece a los niños elementos didácticos y mediante su creatividad, resuelven diversos acertijos.

Como se puede ver en la Figura 1, en el Perú, según la Evaluación Censal de Estudiantes (ECE) del año en la que participaron 542 mil 878 alumnos de segundo grado de Primaria de todo el país, cuyas edades fluctúan entre los siete y ocho años, se evidenció que se ha incrementado la cifra de escolares peruanos que logran resolver problemas matemáticos, en el aula, en un $7.5 \%$ entre el 2015 y el 2016. En el año 2015, de 100 estudiantes del segundo grado de Primaria, 27 lograban resolver un ejercicio matemático; en el año 2016, 34 respondieron correctamente; sin embargo, se ha reducido el número de los que entienden los textos que leen como se puede ver en la Figura 2. Aún sigue siendo un nivel bajo en comparación con otros países y lo que se busca año a año es mejorar estos porcentajes. 
Según la UNICEF (Fondo de las Naciones Unidas parala Infancia), Programa de la Organización de las Naciones Unidas que provee ayuda humanitaria y de desarrollo a niños y madres, en países en desarrollo, el problema educativo más grave que afecta a las niñas y niños de Perú es el bajo nivel existente de comprensión lectora y razonamiento matemático (Unicef, s.f.). Esta afirmación se puede corroborar con el estudio hecho por el Ministerio de Educación sobre el nivel de comprensión de lectura y razonamiento matemático en el Perú (Figuras 1 y 2).

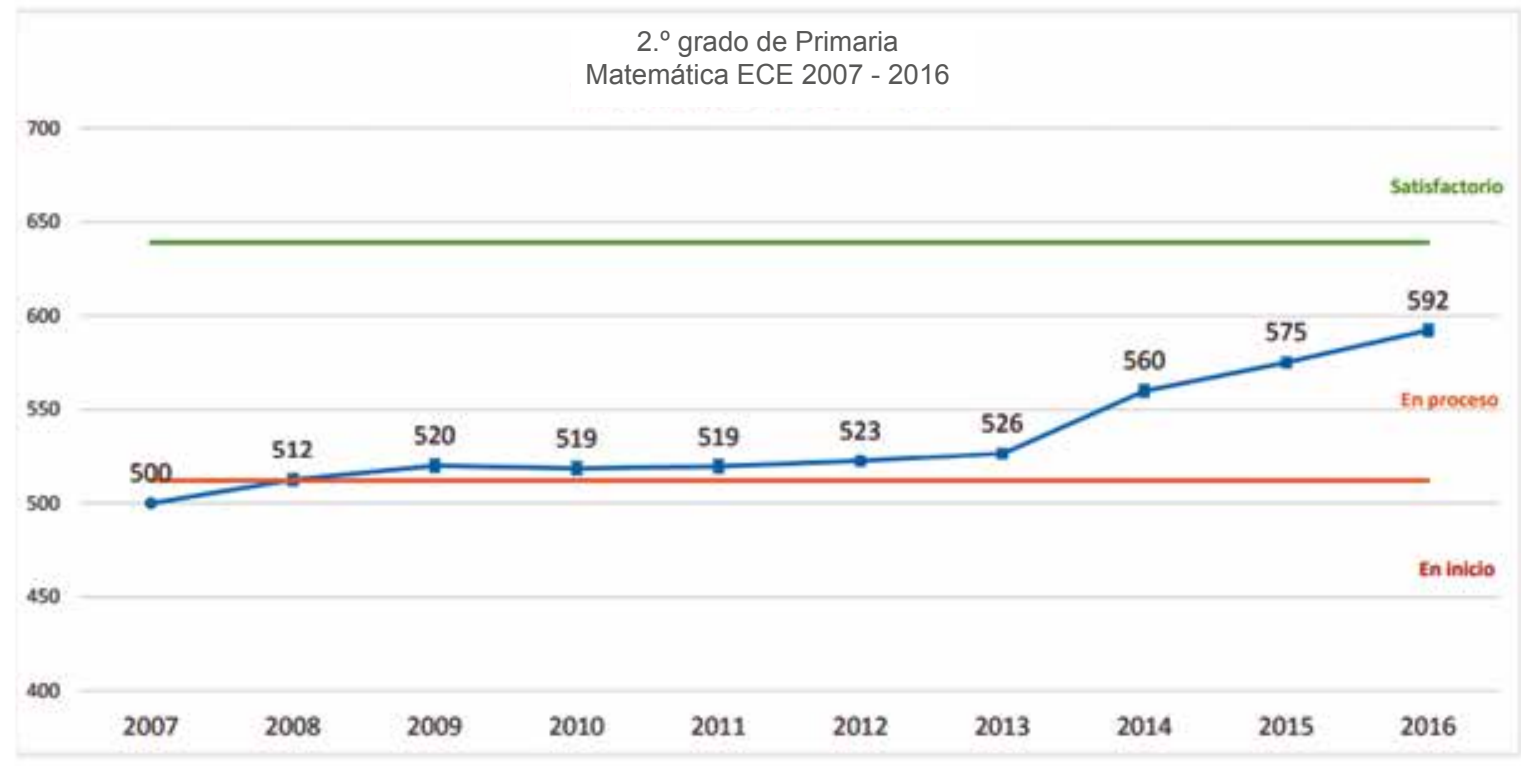

Figura 1. Matemática: resultados nacionales según medida promedio (MINEDU, 2016).

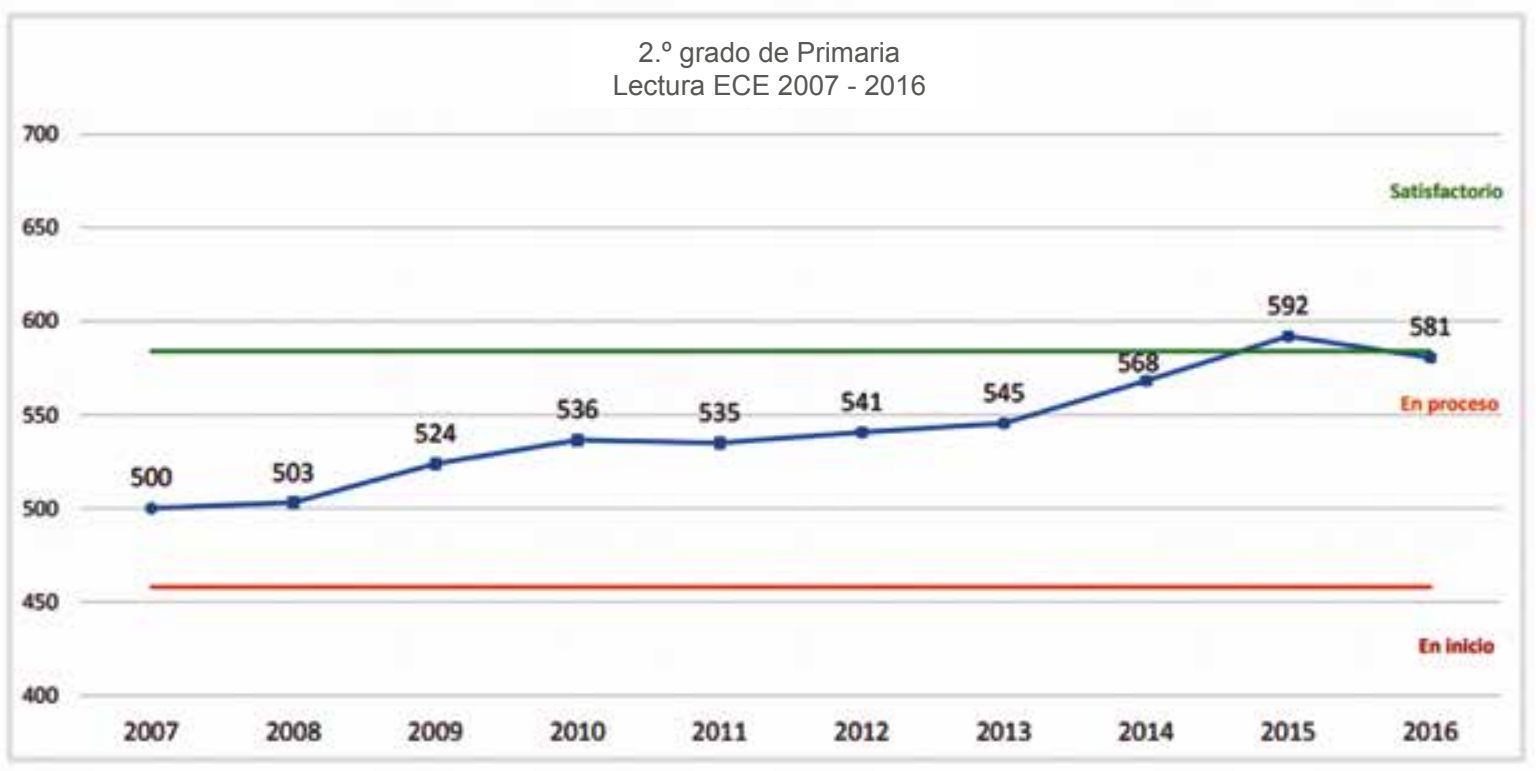

Figura 2. Lectura: resultados nacionales según medida promedio (MINEDU, 2016).

Según el estudio del Ministerio de Educación sobre la comprensión de lectura, los estudiantes del segundo grado de Primaria ubican información que no se encuentra tan fácilmente en el texto que les asignan a leer. Además, deducen ideas 
que les permiten comprender algunas partes específicas del texto, así como entenderlo en su conjunto. Asimismo, aplican las afirmaciones del texto a situaciones nuevas y reflexionan sobre la forma del texto, apoyándose en su conocimiento cotidiano. Estos procesos los realizan en textos de extensión media, estructura simple y complejidad adecuada para el grado (MINEDU, 2016).

A base del estudio hecho por el Ministerio de Educación, en el año 2016, se comprueba que para mejorar, en los niveles de comprensión de lectura y razonamiento matemático, se debe trabajar desde las primeras etapas de la niñez. Los estudiantes que logran el nivel satisfactorio pueden, por ejemplo, deducir el significado de una frase en un texto descriptivo de estructura no compleja adecuada al grado. Esto se debe tener en cuenta cuando se desarrollen las aplicaciones de móviles educativos para nińos con dificultades de aprendizaje.

Actualmente, encontramos diversas aplicaciones educativas en las que a base de juegos, los niños pueden practicar la escritura, lectura y razonamiento matemático de una manera sencilla, divertida e interactiva. Son un soporte de lo que aprenden, en el colegio, y deben ser elegidos según la necesidad de aprendizaje del niño.

Según el Instituto de Estadística e Informática (INEI), la población entre $6-16$ años que hace uso de internet en Lima Metropolitana ha ido en aumento ( Figura 3).

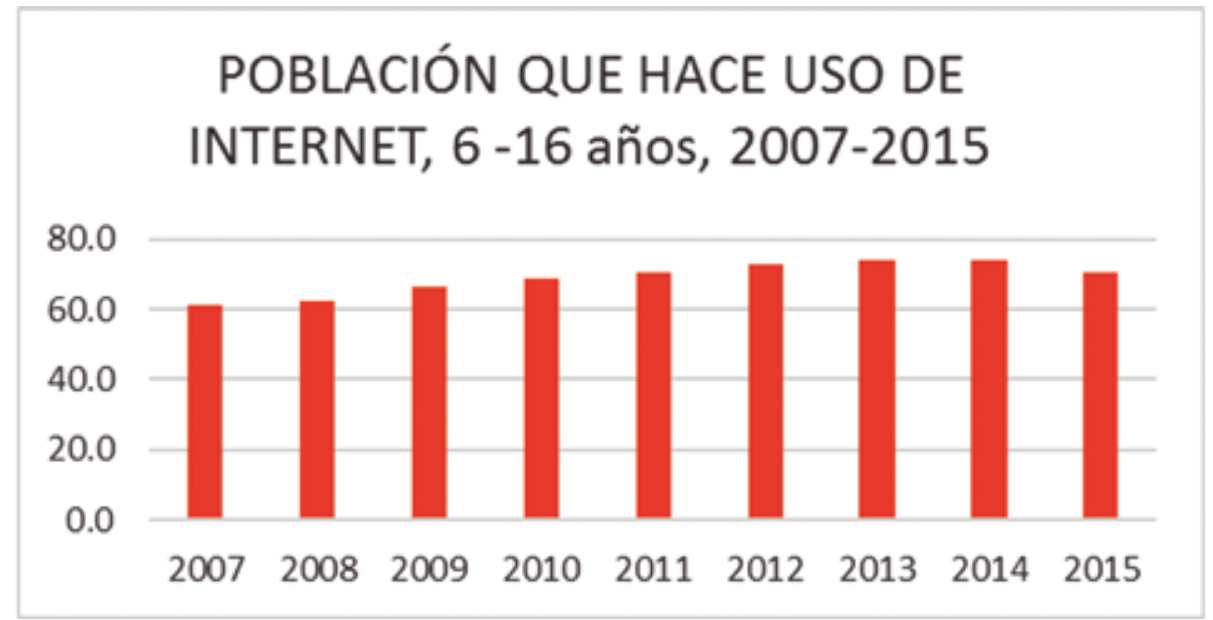

Figura 3. Población que hace uso de Internet (INEI, 2015).

Asimismo, el INEI informó que en el año 2016 el uso de Internet por teléfono celular alcanzó al 17,6\% de la población de seis y más años de edad. Del total de la población de seis y más años de edad que usa Internet, el 17,6\% lo hace exclusivamente por teléfono móvil y una proporción similar $(17,6 \%)$ dentro del hogar. Le siguen el $17,4 \%$ que lo hace solo en cabina pública, el 13,5\% combina el hogar con teléfono móvil, el 7,0\% accede en el hogar y el 2,4\% solo en casa de otra persona, el 1,3\% en el establecimiento educativo y el $20,4 \%$ en dos o más lugares (Instituto Nacional de Estadística e Informática, 2017), lo cual demuestra que un porcentaje considerable accede a internet, a través de un dispositivo móvil y desde su casa. 
En el primer trimestre del año 2017, el $28,2 \%$ dela población de seisy más años de edad accedió a Internet, exclusivamente, por teléfono móvil, cifra superior en 10,2 puntos porcentuales, al compararlo con similar trimestre del año 2016, informó el Instituto Nacional de Estadística e Informática (INEI) mediante el Informe Técnico Estadísticas de las Tecnologías de Información y Comunicación en los Hogares, elaborado con los resultados de la Encuesta Nacional de Hogares (Instituto Nacional de Estadística e Informática, 2017). Este aumento del uso de los dispositivos móviles requiere que enfoquemos al evaluar si los aplicativos de apoyo educativo con los que se cuenta, actualmente, son de utilidad si un niño ingresa desde una Tablet o un Smartphone. Es por eso, que en nuestro estudio, decidimos probarlos en diferentes dispositivos y cuál es el más adecuado según su edad. En la Figura 4, se observa a dos niñas de tres y cinco años haciendo uno de un aplicativo móvil educativo.

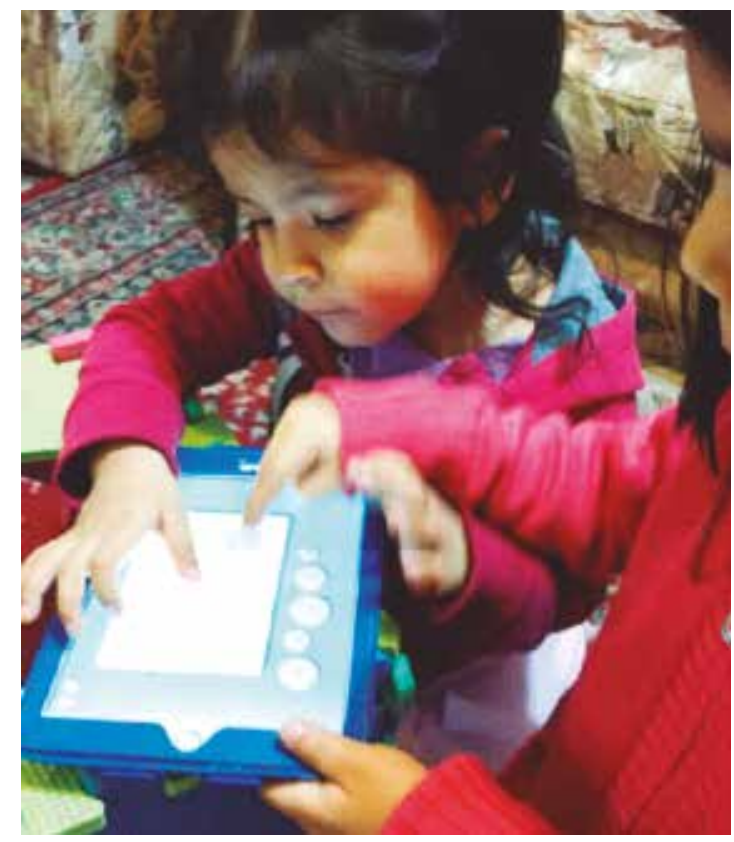

Figura 4. Prueba de aplicativo móvil educativo. Elaboración propia.
En el colegio, los profesores utilizan aplicativos que apoyan el dictado de clases, pero esto también conlleva a que se utilicen más sistemas de control para monitorear las actividades que realicen los nińos y de esa manera, guiarlos para un mejor desempeño.

Los niños, en edad escolar e inicial, tienen diversas dificultades de aprendizaje las cuales son detectadas en el momento que se les está enseńando en la escuela o en casa. En la escuela, los profesores deben estar capacitados para atender estas necesidades de sus alumnos y junto con los padres, analizar de qué manera se mejoraría el aprendizaje de los niños, teniendo como apoyo la tecnología actual. Los padres y maestros son de otra generación e inmigrantes digitales que deben comprender y adaptarse a los cambios en tecnología, así como entender el comportamiento de la generación $\mathrm{Z}$.

Existen diversas aplicaciones, sitios de Internet y métodos que ayudan a los niños con dificultades de aprendizaje y de atención. Son aplicaciones que deben ser desarrolladas de una manera adecuada para que la generación actual las utilice sin inconvenientes. La generación $\mathrm{Z}$ está familiarizada con los juegos y esperan que todo lo que parezca interesante contenga elementos lúdicos (Pérez, 2013).

En "Nativos digitales, inmigrantes digitales" se aborda, en primer lugar, cómo las diferencias entre los alumnos -nativos digitales- y sus profesores inmigrantes digitales- son la causa de muchos de los problemas que afectan a la educación en nuestros días. En segundo lugar, consideramos también la 
alta probabilidad de que el cerebro de los nativos sea fisiológicamente distinto al de los inmigrantes como consecuencia de los estímulos digitales que han recibido a lo largo de su crecimiento (Prensky, Nativos e inmigrantes digitales).

En la presente investigación, se efectuó una evaluación de los aplicativos más utilizados y se analizó la facilidad de uso de los mismos. Estas aplicaciones son desarrolladas bajo el concepto de la Gamificación, en donde tanto los niños como los adultos aprenden jugando. Las características del grupo de niños evaluados son las siguientes:

- A. Tiene siete años. Se le diagnosticó a la edad de cinco, hiperactividad y déficit de atención.

- I. Tiene cinco años. Se le halló dislexia cuando aprendió a leer.

- M, A. de tres años y B, A, S. de seis años fueron diagnosticados con dificultad de aprendizaje.

- Algunas aplicaciones móviles educativas, que pueden utilizar los niños con problemas de aprendizaje para un trabajo conjunto con padres y maestros, según el tipo de dificultad de aprendizaje son las siguientes:

\section{Dificultad para concentrarse e hiperactividad}

a. Classdojo. Es una aplicación muy útil para el estudiante, docente y padre de familia, ya que permite la cooperación. El docente crea el tema a tratar, el estudiante se guía y puede realizar las labores indicadas y el padre puede ir monitoreando qué es lo que viene realizando su hijo en las horas de clases.
Esta aplicación sirve para tener una pauta de lo que se va a enseñar en las horas de clase. Dentro de la app, el padre de familia puede revisar el calendario o línea de tiempo en el cual irá viendo el progreso de lo que su hijo realice, así como los próximos temas a tratar. Además de lo mencionado, también se podrá llevar un control de asistencia a clases. A su vez, es una aplicación muy intuitiva y fácil de usar, ya que está dirigida más para estudiantes del nivel primario, pero también puede utilizarse en el nivel secundario u otros.

b. Readability. Esta aplicación (web y móvil) sirve para gestionar las páginas ya leídas en la web y ubicarlas de una mejor manera, mucho más rápida y eficaz. Organiza lo visto en listas de acuerdo con el interés del usuario; es de fácil acceso y ayuda a la mejora y organización de lo que uno investiga.

c. Google Classroom. Es una aplicación creada, especialmente para la interacción entre el docente y el alumno, ya que gestiona un entorno de trabajo entre ellos. Utiliza las herramientas de Google para facilitar las tareas, tales como el Google Drive, Calendario, entre otras. La función principal del aplicativo es la de crear clases y gestionar la participación de los estudiantes.

d. Icuadernos. Es una aplicación que va dirigida, esencialmente, a los alumnos. Es muy didáctica e intuitiva. $\mathrm{Su}$ aplicación móvil es muy fácil de manejar y posee muchos instrumentos para el aprendizaje del estudiante. En ella, se puede aprender a leer, sumar, restar, otras funciones matemáticas, diferenciar las mayúsculas de las minúsculas, colorear, entre otros. $\mathrm{Su}$ 
interfaz gráfica ayuda a que el estudiante pueda aprender a usarlo de una manera más divertida y sin complicaciones. Su uso es dinámico y ágil, y las variantes que posee ayudan, de una manera sustantiva, al aprendizaje.

\section{Dificultad para leer}

a. For Dyslexia. Es un Startup orientado a brindar apoyo tecnológico a niños con deficiencias en el aprendizaje. Dentro de sus programas, hay varios aplicativos para ayudar, de manera significativa al estudiante. Uno de ellos es Alphabetics, una aplicación diseñada para ayudar a los nińos a reconocer palabras, saber escribirlas correctamente, vocalizarlas de manera correcta, así como, diferenciarlas de otras palabras. Está enfocada en varios niveles, que va de lo más simple o sencillo a lo más complejo.

b. Children's day map. Es una aplicación dirigida a niños para que puedan aprender, de manera divertida, el idioma inglés, ya que pregunta desde la fecha del día presente hasta qué tipo de ropa está usando en ese momento. Su interfaz de usuario es ágil y colorida.

c. Abc Kit Letters. Está dirigido a niños de tres a siete ańos. En ella, los niños pueden aprender, de manera divertida, el abecedario, mejorar su comprensión lectora, diferenciar las palabras, entre otros. Los padres tienen un registro de lo que sus niños han realizado. El entorno de la aplicación es amical y muy bien elaborado lo que ayuda a motivarlos en el aprendizaje. En la Figura 5, la niña está usando el aplicativo mencionado.

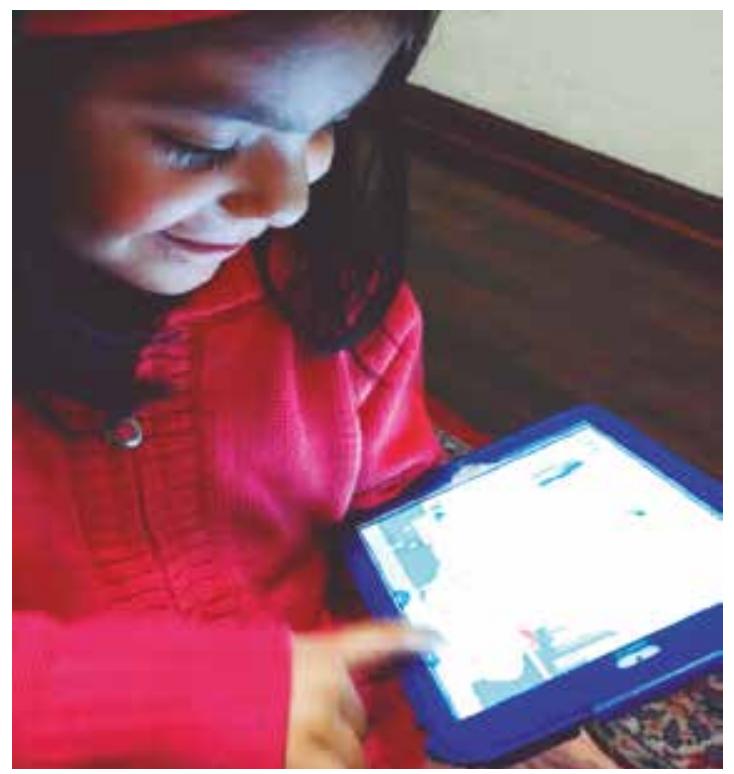

Figura 5. Prueba de aplicativo Abc Kit Letters. Elaboración propia

d. Newsela. Es un aplicativo dirigido a estudiantes para que puedan darle un mejor uso a las lecturas indicadas por el profesor, ya que no solo permite gestionar lo que vaya a leer el alumno, sino que de manera didáctica permite que el docente esté al tanto de lo que el estudiante está leyendo, a hacerles preguntas acerca de lo leído y revisar, de esa forma, si aprendió o no lo indicado en el texto. Su funcionalidad es muy eficaz, ya que el profesor puede llevar un control total de lo que le solicite al alumno. Posee muchos artículos y ha sabido diferenciarse de otros aplicativos con esa funcionalidad.

e. Rave-o. Está dirigido a niños de dos a cuatro años, y su principal función es la de ayudarlos a profundizar más la lectura y correcta alfabetización, para generar así mejores conceptos, conocimientos, ideas, sobre las lecturas revisadas, entre otros. $\mathrm{Su}$ característica principal es la de acelerar el aprendizaje, supervisar las evaluaciones realizadas, a través de informes de manera continua, y mejorar el desempeño estudiantil del habla como de la lectura. 
f. Dytective. Aplicación que permite conocer si el niño o estudiante posee deficiencias de aprendizaje, tanto en la lectura o escritura, ello a través de un test que detecta si existe problema de dislexia en la persona, que le genere dificultades en lo cognitivo. Lo mejor de la aplicación es que con ágiles y dinámicas preguntas ayudan a detectar este problema. Las preguntas van de acuerdo con la edad que aparece en el test. La prueba solo dura 15 minutos, aproximadamente, $y$ en tan solo una hora uno puede conocer el resultado del test aplicado.

g. Geometría Montessori. Aplicación que ayuda al estudiante a familiarizarse, de mejor manera, con el mundo de la geometría, ya que le permite diferenciar las distintas figuras geométricas, con divertidos juegos y más, de una manera fácil y ligera de aprender. Ayuda, de manera eficaz, a asignar un orden jerárquico a las cosas, de manera lógica e intuitiva, y con un entorno gráfico que ayuda, visualmente, a potenciar el conocimiento del niño. En la Figura 6, la niña M. está usando el aplicativo mencionado.

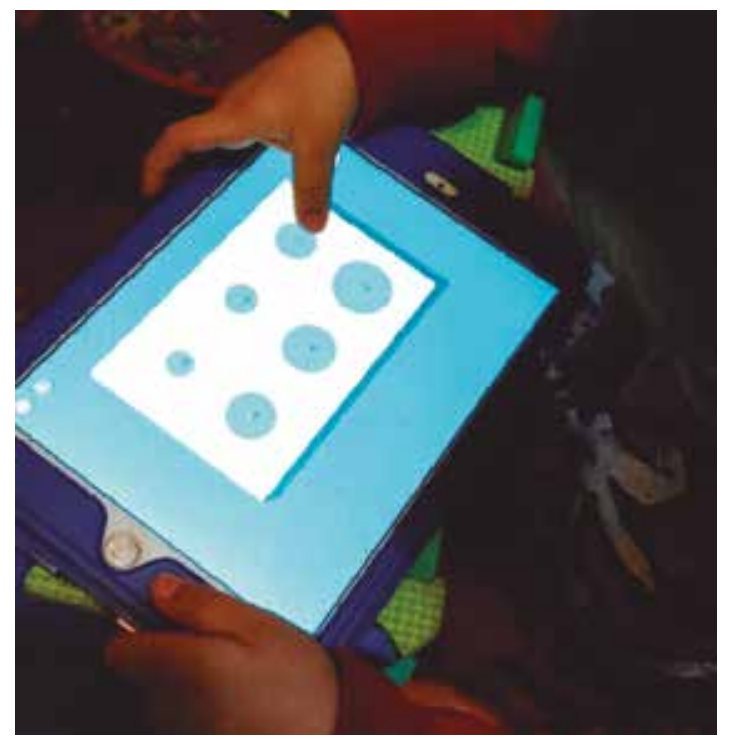

Figura 6. Prueba de aplicativo Geometría Montessori. Elaboración propia

\section{Problemas con las habilidades motoras}

Llevar las bolas al agujero, aplicación dirigida sobre todo a niños con dificultades para la concentración y capacidad motora, ya que aquí, a través del juego de poner las bolas en el agujero, uno puede desarrollar la concentración y la destreza motriz. Contiene una serie de niveles que van aumentando de nivel, según lo vaya avanzando.

\section{Características necesarias para el diseño de aplicaciones para nińos}

El aprendizaje móvil se ha convertido en una de las soluciones de los problemas que confronta el sector educativo. El programa de actividades de la UNESCO se basa en un número cada vez mayor de iniciativas conjuntas, encaminadas a estudiar de qué manera las tecnologías móviles pueden propiciar la consecución de la Educación para Todos (EPT) (Unesco, 2017). Por eso es importante evaluar cuáles son las necesidades que tienen los niños porque son los usuarios que interactúan directamente con los aplicativos, para esto hay que tener en cuenta los aspectos de usabilidad y la comunicación del mensaje, es así como el diseño debe ser lo suficientemente fuerte para mantener su atención. La interfaz debe ser creada según su edad.

Los usuarios tienen diversas necesidades, por ese motivo es necesario que analicemos, primero, cuáles son los objetivos que deseamos lograr y a qué público queremos llegar, ya que a base de ese análisis se establecen las técnicas de usabilidad y la organización del proceso de diseño (Quispe, 2013). Para entender 
las necesidades básicas de los beneficiarios se debe consultar y evaluar mediante encuestas a los profesores y psicólogos para construir aplicaciones que se adapten a las necesidades de los nińos, porque no es lo mismo desarrollar una aplicación móvil para un nińo, que tiene problemas con las matemáticas, a uno que tiene problemas de lectura o atención. Es por ese motivo que, en próximos estudios, se aplicarán pruebas a grupos de usuarios para obtener información concisa sobre las necesidades y requerimientos de los niños, según edades.

Las aplicaciones de aprendizaje móvil se desarrollan utilizando diversas tecnologías y plataformas. Cada implementación tiene características específicas en términos de interfaz de usuario y contenido, e influye en el proceso de desarrollo. Un sistema de aprendizaje móvil consiste en los siguientes componentes: dispositivo de aprendizaje móvil, software de aprendizaje móvil y contenido de aprendizaje móvil (Pocatilu, 2010). El sexo es también una característica importante. A las niñas, les atrae las hadas, princesas y cantantes, mientras que los niños pueden ser atraídos por reyes, autos, guerreros, y héroes. La educación de un niño también es una gran influencia para su personalidad. Su nivel de educación se refleja en sus intereses y preocupaciones, La nacionalidad de los niños es también otra característica que debe ser tomada en cuenta (Tîrziu, 2011). La importancia de la lectura al entender una interfaz debe también considerarse ya que la mayoría los niños menores de seis ańos no sabe leer por lo que es conveniente utilizar botones, iconos, imágenes y sonidos. Los botones deben tener un tamaño adecuado para los dedos de los nińos. Asimismo, es importante destacar que para un niño de tres años le es más fácil utilizar una Tablet que es táctil, a manejar un ratón de una computadora, porque interactúa con ella desde antes de un año y por lo tanto, mientras va creciendo se acostumbra a esa interfaz.

\section{Conclusiones}

Con el uso de aplicaciones móviles educativas para niños con dificultades de aprendizaje los niños van explorando, aprendiendo, descubriendo a través de lo que están realizando con cada uno de los recursos que le proporciona el aplicativo. Además, les posibilita evaluar su propio trabajo y trabajar en equipo.

Muchos niños presentan dificultades de aprendizaje, problemas de concentración y atención, problemas de conducta y dificultades emocionales, que impactan, en su desarrollo educativo, y si no son tratados a tiempo, pueden perjudicarlos durante toda su vida, por eso los padres y maestros juegan un rol importante para que desde el inicio, se puedan detectar y atender estos problemas, apoyándose en la tecnología.

Actualmente, el sistema escolar no se encuentra preparado para acoger a niños con este tipo de problemas. Por ello, hace falta la creación de departamentos especializados para capacitar a los maestros, a través de talleres de inducción, informativos y psicológicos. Otro problema latente es el rechazo a la tecnología por parte de los maestros y padres de familia, que son no migrantes y que necesitan rápidamente integrarse al mundo tecnológico para intervenir en la formación de los niños con las dificultades mencionadas. 
Por tanto, es necesario desarrollar habilidades en niños con problemas de aprendizaje, para ello la tecnología actual y el uso de aplicaciones móviles cobra mayor importancia, ya que los niños son nativos digitales, pertenecientes a una generación que nace con Internet y en consecuencia, el uso de la tecnología es parte de su vida.

Así como, las aplicaciones móviles educativas impulsan la alfabetización y apoyan a aquellos niños con dificultades de aprendizaje, también pueden apoyar a la capacitación de los maestros para mejorar la calidad de la enseñanza.
Las aplicaciones educativas son beneficiosas para los niños porque favorecen procesos de aprendizaje y educación, pero debe entenderse, claramente, que son un apoyo a los métodos tradicionales. Por ello, es necesario el concurso de los padres y maestros para que guien a los niños en el buen uso de estas aplicaciones y así combinarlas en esquemas de aprendizaje que desarrollen todas las habilidades y capacidades físicas e intelectuales.

En próximas investigaciones, se evaluará la importancia de la usabilidad en el diseño de la interfaz de usuario de las aplicaciones móviles educativas.

\section{Referencias}

Aguilera, A. (2003). Introducción a las dificultades de aprendizaje. España: Editorial Interamericana.

Alissa N., Antle, E. \& Cramer, M. (2015). PhonoBlocks: A Tangible System for Supporting Dyslexic Children Learning to Read. TEI International Conference on Tangible, Stanford, USA.

Aparici, R. (2013). Conectados en el Ciberespacio. España: UNE Universidad de Educación a Distancia.

Ardila, A., Rosselli, M. \& Matute, E. (2005). Neuropsicología de los trastornos del aprendizaje. México: UNAM.

Coveñas, R. \& Sánchez, M. (2010). Dislexia: Un enfoque multidisciplinar. España: Gamma.
Federación Estatal de Asociaciones de Profesionales de Atención Temprana. (2000). Libro blanco de la atención temprana (Documentos 55/2005). Espańa: Real Patronato sobre discapacidad.

Instituto Nacional de Estadística e Informática. (2015). Población que hace uso de Internet 20072015. Recuperado de https:// www.inei.gob.pe/media/ MenuRecursivo/indices_ tematicos/cuadro-9.xlsx.

Instituto Nacional de Estadística e Informática. (2017). El 28\% de la población que usa internet lo hace exclusivamente por teléfono móvil.

Kielhofner, G. (2004). Terapia ocupacional: modelo de ocupación 
humana: teoria y aplicación. Argentina: Médica Panamericana.

Kirk, S. (1962). Educating Exceptional Children. California: Wadsworth Publishing Company.

Mainieri, A. \& Méndez, Z. (1986). Detección de problemas de aprendizaje: antología. España: EUNED. Editorial Universidad Estatal a distancia.

Millá, M.G. (2006). Atención temprana de las dificultades de aprendizaje. Rev Neurol, 42, 153-156.

MINEDU. (2006). Resultados de la Evaluación Censal de Estudiantes. Recuperado de http://umc.minedu. gob.pe/resultadosece2016/

Moore, D. \& Jefferson, J. (2005). Manual de psiquiatría médica. España: Elsevier.

Pérez, J. (2013). Gamification: Aprenda a jugar sus cartas digitales. Recuperado de https://www. harvard-deusto.com/gamificationaprenda-a-jugar-sus-cartas-digitales

Pocatilu, P. (2010). Developing Mobile Learning Applications for Android using Web Services. Informatica Economică, 14(3), 106-115.

Prensky, M. (2001). Digital Natives, Digital Immigrants. Madrid, España: Distribuidora SEK S.A.
Quintero, F., Correas, J. \& Quintero, J. (2009). Trastorno por déficit de atención e hiperactividad (TDAH) a lo largo de la vida. España: Masson.

Quispe, A. (octubre, 2013). Diseño de páginas Web universitarias con el apoyo de la Ingeniería de usabilidad. Trabajo presentado en el V congreso Iberoamericano SOCOTE Soporte del Conocimiento con Tecnología, Lima, Perú. Recuperado de http://www.usmp.edu.pe/pdf/ Paper_SOCOTE_2013_Diseno_ de_paginas_Web_universitarias_ con_el_apoyo_de_la_Ingenieria_ de_Usabilidad.pdf

Taylor, R \& Rutter, M. (2002). Child and Adolescent Psychiatry ( $4^{\text {th }}$ ed.). UK: Blackwell.

Tîrziu, G. (2011). Children aimed interfaces for android running devices. Recuperado de ftp://ftp.repec. org/opt/ReDIF/RePEc/rau/jisomg/ SP11/JISOM-SP11-A19.pdf

Torras, E. (2002). Dislexia en el desarrollo psíquico: su psicodinámica. Perú: Grupo Planeta.

Unesco. (2017). Las TIC en la Educación, el aprendizaje móvil. Recuperado de http://www.unesco.org/new/ es/unesco/themes/icts/m4ed/.

UNICEF. (2015). Educación primaria. Recuperado de https://www.unicef. org/peru/spanish/children_3787. html 
